\title{
Heterotrema sarasinorum, eine neue Synascidiengattung aus der Familie der Distomidae.
}

\author{
Von
}

Dr. Karl Fiedler (Zürich).

\section{Hierzu Tafel XXV.}

Unter den Spongien, welche seiner Zeit von den Herren Dr. Dr. Sarasin während ihres Aufenthaltes in Ceylon bei Trincomali (an der Ostküste) gesammelt und vor etwa einem Jahre mir zur Untersuchung übergeben worden waren, fand sich eine Form, die ihrem Ansehen nach durchaus der genannten Thiergruppe anzugehören schien. Sie stellt eine plumpe, unregelmässig verzweigte Masse von ansehnlicher Grösse dar ${ }^{1}$ ), deren einzelne Aeste meist flach zusammengedrückt, gegen das Ende zu jedoch keulenförmig angeschwollen sind. Die Oberfläche zeigt zahlreiche kleine, rundliche Erhabenheiten, welche nur an jenen Stellen, wo das Thier dem Boden aufgelegen haben mag, ganz fehlen und durch Gesteinsfragmente, Bruchstücke von Muschelschalen u. s. w. ersetzt werden. Bezirke der letzteren Art finden sich nur auf einer Seite der Colonie - dass es sich um eine solche handelte, war sofort ersichtlich - und ihre Vertheilung zeigt, dass die Colonie nicht baumförmig aufrecht stand, sondern dem Untergrunde mehr als dickes, nur wenig sich emporhebendes Polster auflag. Die gallertartige Beschaffenheit der ganzen Masse sprach auch nicht gegen die Schwammnatur, da ja solche Spongien ebenfalls bekannt sind.

1) Grösste Länge $81 / 2 \mathrm{~cm}$, mittlere Breite eines Astes $1 \frac{1}{2} \mathrm{~cm}$, grösste Breite $3 \mathrm{~cm}$. 
Schon bei der vorläufigen Untersuchung waren mir weiterhin zahlreiche, gegen die Mitte der Querschnittflächen hin gerichtete wurmartige Bildungen aufgefallen, die dem übrigen Gewebe nur lose einlagerten. Man konnte sie mit der Pincette leicht herausholen, und unter dem Microscop liess sie der erste Blick als ascidien-ähnliche Thiere erkennen. So lag der Gedanke nahe, dieselben möchten sich als Miether in der Spongie eingenistet haben; kommt doch solche, halb parasitäre, halb symbiotische Vergesellschaftung selbst sehr verschiedener Thiere nicht selten vor.

Indessen die microscopische Prüfung von Schnitten, welche durch den vermeintlichen Wirth mitsammt seinen vermeintlichen Gästen gelegt wurden, lehrte alsbald, dass jener nichts anderes ist als die gemeinsame Grundmasse einer coloniebildenden Ascidienart, einer Synascidie, während diese die Einzelthiere oder Ascidiozooiden derselben darstellen. - Wie schwer übrigens die Unterscheidung von Spongien und Synascidien werden kann, namentlich wenn, die Einzelthiere der letzteren nicht gut erhalten sind, das zeigen mehrere, noch aus der neueren Literatur bekannte Fälle thatsächlicher Verwechslung beider Thierformen. So hat erst F. E. SchulzE ${ }^{1}$ ) sicher nachgewiesen, dass die zu den Gummineen gestellte Lacinia stellifica SelenKA's sowie die Cellulophana pileata Oscar SchmidT's keine Schwämme, sondern zusammengesetzte Ascidien sind. So wird man denn auch den Beinamen „spongiforme“, welchen GIARD ${ }^{2}$ ) einer Astellium-(Diplosoma-)Art gegeben hat, zwar für die grosse Aehnlichkeit zwischen Synascidien und Spongien characteristisch finden, weniger aber für die Art selbst.

Ich habe nun die so erkannte Synascidie einer näheren Untersuchung unterworfen und gestatte mir, die erlangten Ergebnisse in Folgendem vorzulegen. Ich schicke voraus, dass es sich um eine neue Form handelt, wie mir nicht nur eigene sorgfältige Vergleichung der Literatur ergab, sondern wie mir auch von einer der ersten Autoritäten auf diesem Gebiete bestätigt wurde. Ich möchte nicht unterlassen, Herrn Prof. Herdman (Liverpool), welcher die grosse Güte hatte, einige meiner Präparate und Zeichnungen zu prüfen, auch an dieser Stelle meinen verbindlichsten Dank für sein so überaus freundliches Entgegenkommen auszusprechen.

1) F. E. Schulze, Unters. über d. Bau u. d. Entwicklg. d. Spongien, III, in: Z. f. wiss. Zool., Bd. 29, p. 92 u. 119 (1877).

2) A. Giard, Rech. sur les Synascidies etc., in: Arch. de Zool. expér. et gén., t. 1, p. 657 (1872). 
Was die Methoden der Untersuchung betrifft, so mussten sich dieselben natürlich auf passende Färbung und Einbettung des conservirten Materials - es stand mir nur eine einzige Colonie zur Verfügung - beschränken. Die Fixirung und Härtung ist, wie mir von den Herren Dr. Dr. SARAsin mitgetheilt wurde, in öfters gewechseltem, starkem Alcohol bewerkstelligt worden. Die Erhaltung der histologischen Einzelheiten erwies sich denn auch im Ganzen als eine recht gute. - Vorsichtiges Isoliren der Einzelthiere mit Nadeln, Pincette, Färbung mit Picrocarmin und Alauncochenille, weniger zweckmässig mit Hämatoxylin, endlich Einschluss in Glyceringelatine, Kanada- oder Tolubalsam liefert brauchbare Uebersichtspräparate. Zur möglichst schonenden, Schrumpfungen ausschliessenden Entwässerung der zum Einlegen in Balsam bestimmten Präparate bediente ich mich entweder des Schulze'schen Entwässerungs-Apparates oder eines einfachen Verfahrens, das mir von Herrn Dr. OverTon mitgetheilt wurde; dasselbe besteht darin, die zu entwässernden Gegenstände in einer Schale mit schwachem Alcohol innerhalb einer grösseren, gut schliessenden Dose, welche mit starkem Alcohol gefüllt ist, aufzustellen; allmählich erhalten beide Alcoholmengen denselben Concentrationsgrad, und man kann nun das Verfahren bis zur Erreichung des gewünschten Zieles wiederholen.

Den in Benzol gelösten Tolubalsam sowohl wie das nach einer neuen Formel hergestellte Picrocarmin verdanke ich der Freundlichkeit von Herrn Kantonsapotheker KelLer. Beides hat sich auch für meine Zwecke bewährt. Der Tolubalsam hellt noch stärker auf als Canadabalsam, was bei gut gefärbten Uebersichtspräparaten zur deutlichen Erkennung der übereinandergelagerten Organsysteme von grossem Nutzen ist.

Zur Anfertigung von Schnittpräparaten war Einbettung in Paraffin vor derjenigen in Celloidin $\mathrm{zu}$ bevorzugen. Es wurden sowohl von Stücken der ganzen Colonie wie von isolirten Einzelthieren Schnittserien gemacht, wobei ein ScHIEFFERDEckeR'sches Microtom neuester Construction zur Verwendung kam und Schnittdicken von $1 / 10$ bis $1 / 10_{0} \mathrm{~mm}$ benutzt wurden. Das Aufkleben der Schnitte auf Deckgläschen oder dünne Glimmerstreifen geschieht mit Vortheil in der Weise, dass sie mittels Pinsels mit einer feinen und darum rasch trocknenden Schicht sehr dünnflüssigen Collodiums überzogen werden. Oft gelingt es nachher, die durch die Collodiumhaut vereinigten Schnitte in ihrer Gesammtheit vom Deckglas abzuziehen, worauf man sehr leicht und rasch zu färben im Stande ist. Ebenso sicher, nur 
etwas langsamer, gelingen die Färbungen natürlich, wenn die Schnitte auf dem Deckglas belassen werden müssen. Neben dem schon erwähnten Picrocarmin verwendete ich zur Schnittfärbung noch Hämatoxylin (nach Delafield), combinirt mit Eosin, sowie Alauncochenille (nach Czоков). Letztere diente auch zur Durchfärbung ganzer Stücke.

Mein optischer Apparat bestand aus den trefflichen REICHERT'schen Systemen $1^{\mathrm{a}}, 4,8^{\mathrm{a}}$ und der homogenen Immersion $\left.{ }^{1}\right|_{20}$ ". Sämmtliche Zeichnungen sind mit dem ABBE'schen Zeichenapparat entworfen, mit dessen Hilfe auch gleichzeitig die Vergrösserungsziffern bestimmt wurden.

In der Art der Behandlung schliesse ich mich im Wesentlichen

- der von Herdman vorgeschlagenen Reihenfolge an ${ }^{1}$ ) und werde nach Beschreibung der Organsysteme und auf Grund derselben endlich die Frage nach der systematischen Stellung der neuen Form zu erörtern haben.

A e ussere Form der Colonie. Wie schon in den einleitenden Bemerkungen hervorgehoben wurde, erinnert die Colonie in ihrer Form sehr an eine Spongie. Der plumpe Körper ist von gallertartiger Consistenz und entsendet eine Anzahl kurzer, gegen das Ende leicht keulenförmig anschwellender Fortsätze (Fig. 1). Dieselben erheben sich einerseits nur wenig über die Unterlage, berühren sie jedoch andrerseits nur an ein paar Orten unmittelbar. In der nächsten Umgebung dieser, schon durch die Einlagerungen von harten Fremdtheilchen gekennzeichneten Stellen fehlen die Ascidiozooiden so gut wie vollständig. Im Uebrigen unterscheidet sich die „Unterseite“ in nichts von der "Oberseite“. Hier wie dort stehen die kleinen Einzelthiere so dicht gedrängt, dass die gemeinsame Grundmasse eine fast wabenartige Beschaffenheit erhält. Hier wie dort sind sie senkrecht zur Oberfläche der Colonie gerichtet, und ein Querschnitt zeigt daher stets das in Fig. 2 wiedergegebene Bild.

Dadurch, dass der vorderste Theil des Körpers der Einzelthiere ein wenig über die allgemeine Begrenzungsfläche hervorragt, sind die Einzelthiere leicht zu erkennen und auf ihre Vertheilung zu prüfen. Anfänglich scheinen sie ganz regellos zerstreut. Bei genauer Be-

1) W. A. Herdman, Report on the Tunicatae, part I, Ascidiae simplices, in: Challenger-Reports, vol. 6 (1882); part II, Ascidiae compositae, in: Challenger-Reports, vol. 19 (1886). 
trachtung findet man einige Stellen von verschieden grosser Ausdehnung, wo sie doch zu bestimmten Systemen geordnet sind. Die Systeme zählen meist 5-10 Einzelthiere, welche die Ecken eines entsprechenden Polygons einnehmen, während seine Seiten durch seichte Furchen vertreten sind, die zugleich das System von den benachbarten abgrenzen. Auch jene Stellen der Oberfläche, wo die Einzelthiere keine Systeme bilden oder die Systeme wenigstens verwischt scheinen, weisen jene Furchen auf, wenn dieselben auch weniger scharf gezogen sind und nicht so regelmässig zusammenhängen (vergl. Fig. 1). Alles in allem genommen, wird zu sagen sein, dass die Ascidiozooiden zu meist undeutlichen, an beschränkten Stellen jedoch durch Furchen ziemlich scharf abgegrenzten und dann etwa 5-10-gliedrigen Systemen vereinigt sind.

Nach Analogie anderer Arten wäre zu erwarten, dass die Mitte jedes Systems durch einen für die Glieder desselben gemeinsamen Cloakenraum eingenommen würde. Ein solcher bezw. seine Oeffnung lässt sich nun zwar weder bei der Oberflächenbetrachtung noch auf Querschnitten wirklich erkennen, und ganz unzweifelhaft münden die Ausfuhröffnungen mancher Einzelthiere unmittelbar nach aussen; aber es ist $\mathrm{zu}$ bedenken, dass bei der Conservirung durch Zusammenziehung der gemeinsamen Grundmasse etwa vorhandene Cloakenöffnungen leicht zum Verschwinden gebracht werden konnten. Für ihre ursprüngliche Existenz spricht der Umstand, dass die weiterhin $\mathrm{zu}$ beschreibenden Analanhänge der Einzelthiere sehr oft auf einen Punkt hin convergiren und in ebenso gerichtete, dicht unter der Oberfläche verlaufende Canäle hineinragen. - Ueber die Färbung der Colonie kann ich leider keine Angaben machen. Ihre jetzige Farbe ist das gewöhnliche Graubraun älterer Spirituspräparate, doch weiss man ja, dass das oft sehr lebhafte Colorit vieler Synascidien gerade durch Alcohol bald zerstört wird.

Der gemeinsame Mantel. Die Masse, welche die Einzelthiere einer zusammengesetzten Ascidie vereinigt, entspricht bekanntlich dem Cellulose-Mantel oder der Testa (Tunica externa) der einfachen Ascidien in jeder Hinsicht. Diese Masse besteht einerseits aus einer Grundsubstanz, von welcher zuerst 0 . Hertwig ${ }^{1}$ ) für die einfachen

1) O. Hertwig, Unters. über d. Bau u. d. Entwicklung des CelluloseMantels d. Tunicaten, in: Jen. Zeitschr. f. Naturw. u. Med., Bd. 7 (1873). 
Ascidien, später DeLla $V_{A L L E}{ }^{1}$ ) auch für die zusammengesetzten nachwies, dass sie ursprünglich eine Cuticularbildung der Epidermiszellen ist, andrerseits aus zahlreichen Zellen, welche nachträglich von der Epidermis aus in die Grundsubstanz eingewandert sind. Grundsubstanz und Zellen bilden sich nun bei den einzelnen Gruppen in verschiedener Weise aus. Sind bei den einfachen Ascidien sehr oft zwei Arten von Zellen vertreten, nämlich meist spindelförmige, bindegewebsartige und merkwürdig umgewandelte, sog. Hohl-, Blasen- oder Kugelzellen, so mangeln die letzteren bei den zusammengesetzten Ascidien in der Mehrzahl der Fälle ganz, in anderen sind sie nur sehr spärlich entwickelt. Auch bei unserer Form gelangen sie nur selten und dann immer vereinzelt zur Wahrnehmung, jedoch stets in ihrer characteristischen Ausbildung (Fig. 13). Dagegen lagern die kleinen, gewöhnlichen Bindegewebszellen ähnlichen Zellen der Grundmasse zahlreich ein und bilden auch da und dort dichtere Anhäufungen. Ihrer Fähigkeit amöboider Gestaltsveränderung entsprechend, bieten sie die verschiedensten Formen dar, von der vollkommen abgerundeten bis zur mehrfach verästelten. Pigmentführende Zellen konnten nicht nachgewiesen werden.

Die Grundsubstanz ist völlig hyalin und schliesst sich darin dem bei den Synascidien vorherrschenden Verhalten an, während bei den einfachen Ascidien bekanntlich fibrilläre Streifung keine Seltenheit ist. Kalkkörper von characteristischer Form kommen ihr nicht zu. Dagegen sieht man allerlei, oft von einer dichten Zellschicht umgebene, zufällig umschlossene Fremdkörper, wie kleinste Steinchen, Spongiennadeln, Gruppen von Diatomeen u. s. w. Immerhin ist ihre Menge nicht so bedeutend, um selbst bei ziemlich dicken Querschnitten die durchscheinende Beschaffenheit der Grundsubstanz merklich zu beeinträchtigen. Auch bei microscopischer Durchmusterung fällt eine andere Bildung sofort weit mehr auf. Es sind ansehnliche, kreisförmig umschriebene Massen, welche sich im Innern der Colonie in grösserer Zahl finden als in den Randtheilen. (In Fig. 3 sind einige derselben bei schwacher Vergrösserung angedeutet.) Ihrer Natur nach gehören sie verschiedenen Gruppen an. Einige bestehen aus blossen Anhäufungen einer körnigen Substanz, welche nach DeLLA VALLE ${ }^{2}$ ) von epithelialen Zellen abstammt. Andere stellen junge Knospen dar, deren Ent-

1) Della Valte, Rech. sur l'anat. des Ascidies composées, in: Arch. Ital. de Biologie, t. 2 (1882).

2) 1. c. p. 11 . 
wicklungsgang sich aber nicht näher verfolgen liess, da alle auf ungefähr gleicher Stufe der Ausbildung standen. Noch andere entsprechen offenbar den Querschnitten reiner Muskelfortsätze des Muskelmantels der Einzelthiere und dienen dazu, die Einzelthiere in den gemeinsamen Cellulose-Mantel zurückzuziehen, ein Verhalten, das auch schon früher beschrieben worden ist, so von Herdman bei Leptoclinum moseleyi und L. thomsoni ${ }^{1}$ ).

Eigentliche „Gefässe“, die ja ihrer Entstehung nach auch auf die Ectodermschicht der Einzelthiere zurückzuführen sind (HerTwiG) ${ }^{2}$ ), fand ich nur wenige und immer nur in der Nähe der Oberfläche der Colonie (Fig. $3 G$ ); sie weisen gewöhnlich eine schwache Anschwellung ihres Endtheiles auf. Bei anderen Arten, Botrylloides z. B., sind reich und eigenartig entwickelte Gefässnetze bekannt. Mit solchen Gefässen dürfen die grossen Hohlräume nicht verwechselt werden, welche an vielen Stellen, ebenfalls nahe der Oberfläche, vorkommen. Schon bei dem Versuche, Einzelthiere zu isoliren, fällt es auf, dass die oberste Schicht des gemeinsamen Mantels sich leicht als dünnes Häutchen abziehen lässt. Das rührt nicht etwa von einer durch verstärkte Einlagerung von Fremdkörpern oder sonstwie erhöhten Consistenz her, wie bei einigen anderen Gattungen; es erklärt sich vielmehr aus dem Vorhandensein eben jener Spalträume unter der obersten, etwa ${ }^{1} / 5 \mathrm{~mm}$ dicken Schicht (Fig. 3). Auf Querschnitten constatirt man, dass die Spalträume oft die Ausfuhröffnungen zweier Einzelthiere in Verbindung setzen, und obwohl mir, wie bemerkt (S. 863), unmittelbar beweisende Beobachtungen mangeln, scheint es mir wahrscheinlich, dass die von den Thieren eines Systems ausgehenden Spalträume ungefähr in der Mitte desselben in eine gemeinschaftliche Cloake münden können.

Die Einzelthiere. Ueber die Stellung der Einzelthiere oder Ascidiozooiden in der gemeinsamen Mantelmasse und ihre Gruppirung zu Systemen wurde das Wesentliche bereits in den vorangehenden Abschnitten mitgetheilt. Hier sei nur noch hinzugefügt, dass die auffallend lose Einlagerung der Ascidiozooiden wohl auf eine die $\mathrm{Zu}-$ sammenziehung der Grundmasse noch übertreffende Contraction der Einzelthiere bei der Conservirung zurückzuführen ist. Wenigstens berühren bei lebenden Synascidien die Ascidiozooiden die Grundmasse

1) Herdman, 1. c., part II, p. 272 u. 290, pl. XXXVII, fig. 16, u. pl. XL, fig. 5 .

2) Hertwig, l, c. p. 53. 
mit ihrer ganzen Oberfläche auf's engste. An drei Stellen ist aber auch bei unserer Form die Verbindung stets erhalten: an den beiden Oeffnungen und an der Basis der Einzelthiere. An der Mund- oder besser Einfuhröffnung wird dieselbe dadurch hergestellt, dass die gemeinsame Mantelmasse sich noch eine ganze Strecke weit auf die Innenseite des Einfuhrtrichters fortsetzt, sich gleichsam auf und in denselben umschlägt. O. HeRTwig hat diesen Zusammenhang zuerst bei den "einfachen Ascidien aufgedeckt. Bei der Ausfuhröffnung wird die Verbindung besonders durch die drei Analanhänge (Fig. 3 u. $4 A A$ ) oder-zungen vermittelt, welche sich hier meist in die Grundmasse einsenken. Manchmal sah ich den einen oder andern dieser Anhänge

" frei in einen jener oberflächlichen Spalträume hineinragen (p. 865), was der Vermuthung Raum lässt, die Analanhänge könnten durch Bewegung in bestimmter Richtung bei der Weiterbeförderung der durch die Analöffnung ausgestossenen Nahrungsreste betheiligt sein. Endlich erfolgt die Verbindung mit der Grundmasse durch die Muskelfortsätze, welche schon kurz erwähnt wurden (p. 865) und in Verbindung mit der übrigen Musculatur näher zu besprechen sein werden (vergl. auch Fig. $4 M F$ ).

Der Körper jedes Einzelthieres (Fig. 4) zerfällt durch eine ziemlich tiefe Einschnürung in zwei ungleich grosse Theile. Unsere Form würde demnach der Milne Edwards'schen Gruppe der „Didemniens“ angehören ${ }^{1}$ ). Die Länge des oberen Abschnittes, welcher besonders den Kiemensack enthält, beträgt $\left.1^{1}\right|_{2}-2 \mathrm{~mm}$, die Länge des unteren, welcher namentlich den Verdauungs- und Geschlechtsapparat umschliesst und daher als Eingeweidesack bezeichnet wird, $\left.{ }^{1}\right|_{2}-1 \mathrm{~mm}$. Sonach schwankt die Gesammtlänge der Einzelthiere zwischen $2-3 \mathrm{~mm}$. $\mathrm{Zu}$ bemerken ist noch, dass der untere Abschnitt nicht immer in der Längsrichtung des oberen liegt, sondern demselben bisweilen etwas zugeknickt ist.

Der Mantel der Einzelthiere (Muskelmantel). Den Mantel bedeckt bei allen Ascidien zu äusserst eine dünne ectodermatische Membran. Sie besteht aus stark abgeplatteten, mit deutlichen Kernen versehenen Zellen, welche ähnlich auch bei unserer Form die nur spärlich vorhandenen Gefässfortsätze auskleiden. Der hervorstechendste

1) H. Minne-Edwands, Observ. sur les ascidies des côtes de la Manche. Paris, in: Mém. Acad. Sc., t. 18, p. 217 (1842). 
Bestandtheil des im Uebrigen bindegewebigen Mantels ist die (glatte) Musculatur. Dieselbe tritt hier nur in Längsbändern auf, Querbänder fehlen, die Ein- und Ausfuhröffnungen ausgenommen, welche beide von deutlichen Ringmuskellagen umgeben sind (Fig. 4 bei $E$. Oe u. A. Oe).

Was zunächst die Längsbänder betrifft, so sind deren am vorderen Körperabschnitt 20-30 an Zahl, und in jedem Band liegen 2-4-6 lange, glatte Muskelfasern neben einander (in Fig. 4 sind nur die Bänder, in Fig. 5 auch die Fasern dargestellt). Auf den unteren Körperabschnitt, den Eingeweidesack, gehen zwei breite, seitliche Muskelbänder über (Fig. $4 M F$ ), deren Fasern z. Th. von vereinigten Fasern des Oberkörpers stammen, z. Th. von einem ebenfalls ziemlich breiten Muskelband herrühren, welches zwischen Kiemensack und Peribranchialraum unter der Reihe der Languets (Fig. $4 L$ ) verläuft. Zweigen sich schon auf dem Kiemensack gelegentlich einzelne Fasern von einem Strange ab, um unter spitzen Winkeln zu einem anderen an- und überzusteigen, so wird in der Nähe des Branchial- oder Einfuhrtrichters und noch mehr in den Lappen desselben die Auflösung in getrennte und sich mehrfach kreuzende Fäden vollständig. Dieser Branchialtrichter besteht aus einem hohlcylindrischen Grundtheil von ansehnlicher Höhe, an welchen sich sechs, je nach dem Contractionszustand mehr zugespitzte oder mehr abgerundete kurze Zipfel anschliessen, wo sich die Längsmuskelfasern in der in Fig. 5 wiedergegebenen Weise vertheilen. Der Basaltheil dagegen ist von einer starken Ringmuskelschicht umgeben, die aus mehreren Dutzend über einander geschichteten Fasern besteht. Ungefähr ebenso stark ist der Sphincter der Ausfuhr- oder Analöffnung, welche seitlich von der Branchialöffnung, jedoch erheblich tiefer als diese, etwas über der mittleren Höhe des vorderen Körperabschnittes, liegt (Fig. $4 A O e$ ). Sie bildet einen in die Länge gezogenen Schlitz, welcher - wie mit Rücksicht auf die systematische Stellung unserer Form besonders betont sei - eine einfache Begrenzungslinie besitzt und nicht in Zipfel ausgezogen ist. Dagegen wird sie überdeckt von einem dreitheiligen Fortsatz des Mantels, dem bereits erwähnten Analanhang (Fig. $4 A A$ ), dessen Zipfel etwa doppelt so lang, aber nicht ganz so breit sind, wie diejenigen des Branchialtrichters.

Die Tentakel. Am Grund des Branchialtrichters, kurz bevor sich derselbe zum Kiemensack erweitert, findet sich bei allen Synascidien eine Gruppe von Tentakeln. Bei unserer Form sind es deren 16, und zwar stehen sie in zwei Kreisen zu je 8 (Fig. 4 T). Die 
grösseren Tentakel nehmen den oberen Kreis ein, sitzen mit breiter Basis auf und verschmälern sich rasch zu engeren Schläuchen, welche nach abwärts hängen. Die kleineren stehen etwas über und zwischen den grösseren und sind sehr kurz, fast bloss papillenförmig. Beiderlei Tentakel sind hohl.

Der Ki e men s a ck. Der Kiemensack gehört sowohl physiologisch als morphologisch zu den wichtigsten Bestandtheilen des Ascidienkörpers: physiologisch, wegen seiner Bedeutung als Athmungsorgan einerseits und als Anfangstheil des Verdauungsapparates andrerseits, - morphologisch, weil er in seiner Ausbildung mancherlei Unterschiede aufweist, welche zur Characterisirung der systematischen Gruppen wesentlich beitragen.

In der vorliegenden Form ist der Kiemensack sehr schön entwickelt. Seine Grösse verhält sich zu derjenigen des Eingeweidesackes wie 2:1 oder 3:2. Er ist von 8-10, bei einigen Exemplaren sogar von 12 Querreihen von Kiemenöffnungen durchsetzt, und jede Reihe enthält 9-10 Stigmata jederseits zwischen Endostyl und Languets. Die mittleren Oeffnungen haben die Gestalt länglicher Rechtecke, deren Ecken freilich abgerundet sind (Länge etwa $0,1 \mathrm{~mm}$, Breite 0,03-0,05 mm). Nach den Seiten, besonders nach dem Endostyl zu, werden sie nicht nur kleiner, sondern gehen auch in andere, fast dreieckige Formen über (vergl. Fig. 4). Die Flächenstreifen zwischen den Kiemenreihen besitzen eine Breite von 0,03-0,04 mm, diejenigen zwischen den einzelnen Kiemenöffnungen eine solche von $0,01-0,03 \mathrm{~mm}$. Die Wimperzellen selbst, welche alle diese Rahmen auskleiden, sind lang gestreckt, etwa $0,007-0,01 \mathrm{~mm}$ breite und $0,005 \mathrm{~mm}$ hohe Zellen mit entsprechend geformtem deutlichen Kern. Die Länge ihrer dicht gestellten und meist in vollkommener Weise erhaltenen Wimpern beträgt bis $0,007 \mathrm{~mm}$ (Fig. 14).

Von den Gefässsystemen, welche den Kiemensack durchsetzen, ist nur dasjenige der Quergefässe deutlich ausgebildet. Dieselben verlaufen in der Mitte zwischen den Reihen der Kiemenöffnungen. Sie sind an gut gefärbten Präparaten als förmlich nach innen vorspringende Leisten sichtbar (auch in Fig. 4 angedeutet). Sowohl vor dem Eintritt in den stärkeren ventralen als vor der Mündung in den etwas schwächeren dorsalen Hauptlängsstamm erweitern sie sich etwas; besondere Anhänge, die wir von einigen anderen Arten kennen, finden sich hier nicht. 
Der Endostyl. So verschiedenartig der Kiemensack selbst bei den verschiedenen Arten gebaut ist, so gleichartig - wenigstens in Bezug auf die allgemein auffallenden Verhältnisse - sieht jenes merkwürdige, als Endostyl oder Bauchrinne bezeichnete Organ aus, welches ihn seiner ganzen Länge nach auf der Ventralseite durchzieht (Fig. 4 E). Dem Vorkommen nach schon lange bekannt, ist seine Bedeutung als Schleimdrüse und damit seine physiologische Function bei der Nahrungsaufnahme erst durch FoL ${ }^{1}$ ) nachgewiesen worden. Was seinen feineren histologischen Bau bei den Synascidien betrifft, so hat Della VALle ${ }^{2}$ ) davon kürzlich eine eingehende Darstellung geliefert, der ich nichts Neues hinzuzufügen habe. Ich beschränke mich also auf Angabe besonderer Formverhältnisse bei der untersuchten Art.

Bei den meisten Einzelthieren verläuft der Endostyl fast völlig gerade. Der wellig gebogene, manchmal bis zur förmlichen Fältelung gehende Verlauf, den er bei einigen wenigen Exemplaren aufwies, dürfte somit bei unserer Art eine Contractionserscheinung sein, während dies bei anderen Arten nach mehrfachen Angaben zum normalen Verhalten werden kann. Die Breite des Organs beträgt fast $1 /{ }_{10} \mathrm{~mm}$. Gegen hinten und ebenso gegen vorne zu, wo sich der Endostyl in schön geschwungenem, ,,gewölbeartigem" Bogen zu dem Peripharyngealband begiebt, welches gleich unterhalb des Tentakelkranzes liegt, verschmälert er sich etwas und endet mit einer kleinen durch eine Einsenkung unterbrochenen Vorwölbung (vergl. Fig. $5 E$ ). Die hell und dunkel erscheinenden Bänder der Flächenansicht (Fig. $4 E$ u. Fig. 11) entsprechen den mit verschieden hohen Zellen bepflasterten Abstufungen und Terrassen des Endostyls, welche der Querschnitt aufweist (Fig. 8-10). Der Querschnitt zeigt übrigens ein Bild, das in seinen Einzelheiten von den mir bekannten Zeichnungen des betreffenden Organs anderer Synascidien mehrfach abweicht. Es bestätigt dies die Angaben Della VALLE's und lässt erwarten, dass bei genauerer Untersuchung noch mehr Gattungs- oder selbst Artunterschiede auch am Endostyl auffindbar sein werden. Ich gebe zum Vergleich neben meiner Figur 10 in den Fig. 8 und 9 Querschnitte durch das Endostyl von Polycyclus renieri ${ }^{3}$ ) und Botrylloides perspicuum ${ }^{4}$ ). Die einander entsprechenden Theile sind so leicht erkennbar, dass weitere Beschreibung überflüssig

1) Fou, Ueber die Schleimdrüse oder den Endostyl der Tunicaten, in: Morph. Jahrb., Bd. 1, p. 223 (1874):

2) Della Valie, 1. c. p. 18 ff. (1882).

3) Della Valle, 1. c. T. ПI, Fig. 14.

4) Herduan, l. c. part II, T. III, Fig. 5. 
sein dürfte. Nur auf die im Verhältniss zur Gesammthöhe grosse Breite des Organs bei unserer Art gegenüber den beiden anderen Arten sei besonders aufmerksam gemacht. Die langen Cilien in der Mitte des eigentlichen Drüsentheils zeigten sich deutlich auf mehreren Schnitten, die kürzeren Wimpern dagegen, die sonst auf den Seitentheilen auftreten, waren nicht zu sehen.

Oefters stand mit dem Endostyl eine merkwürdige Bildung in Zusammenhang, die sonst nicht erwähnt wird. Es sind zwei Reihen von rundlichen Blasen, welche, in der Längsrichtung regelmässige $\mathrm{Ab}$ stände einhaltend, einander paarweise gegenüberliegen. Dem Aussehen und der Grösse nach ähneln sie den hellen, blasenförmigen Zellen, die in grösserer Menge besonders vor der Einschnürungsstelle zwischen Kiemen- und Eingeweidesack in letzterem auftreten, sich aber von da mehr vereinzelt sowohl nach unten wie nach oben verbreiten (Fig. $4 N$ ? u. Fig. 11). Da man bei vielen einfachen Ascidien Harnsäure abscheidende Organe nur in Form von ähnlichen, ebenfalls die Eingeweide umgebenden Zellanhäufungen findet, so ist wohl anzunehmen, dass es sich auch hier um entsprechende Function dieser characteristischen Zellen handelt. Ihre Ausdehnung auf die Region des Kiemensackes und ihre morphologisch interessante Anordnung neben dem Endostyl würden natürlich dieser Deutung wenigstens nicht entgegen sein. Die dichte Umhüllung der Zellen mit zahlreichen, wohl Gefässen angehörigen Kernen spricht dafür. Andrerseits fehlt hier freilich der Nachweis des Harnsäuregehaltes noch, welcher bei den einfachen Ascidien erbracht ist.

Peripharyngealbänder und Languets. Die Peripharyngealbänder, welche, stets von den Seitenwänden des Endostyls ausgehend, die Basis der Einfuhröffnung nahe unter dem Tentakelkranz umgreifen und sich dorsal vereinigen, bieten keinen Anlass zu besonderen Bemerkungen. Sie sind ziemlich breit und tragen auf der nach oben gekehrten Zellreihe kurze Wimpern. Sie stellen nach der jetzigen Auffassung den Weg dar, auf welchem der vom Endostyl abgesonderte Schleim in die Nähe der Einfuhröffnung gelangt, wo sich eingestrudelte Nahrungstheilchen mit ihm mischen und nun, sei es durch den Wasserstrom des Kiemensackes, sei es unter Betheiligung der Wimpern der Lamina dorsalis bezw. der stellvertretenden „Languets“, zur eigentlichen Mundöffnung am Grund des Kiemensackes geführt werden (vergl. Fig. 4, $P B, L, O e O e$ ).

Der dorsale Vereinigungspunkt der beiden Bänder ist auch hier 
eigenartig gestaltet, zu einem „Dorsaltuberkel“, „Wimperorgan“, „,Riechtuberkel", umgebildet, das seiner Beziehung zu Ganglion und Neuraldrüse wegen dort beschrieben werden soll.

Die Reihe der „Languets“ (Fig. 4, $L$ ) vertritt auch bei dieser Art, wie bei der Mehrzahl der Synascidien, den Platz einer eigentlichen Lamina dorsalis, welche den meisten einfachen Ascidien zukommt. Die Languets stehen als fingerförmige Fortsätze jeweilen einzeln zwischen den Reihen der Kiemenöffnungen und ragen bis auf etwa ein Drittel des Querdurchmessers der Kiemenhöhle in diese hinein. Ihr etwas verdicktes Ende ist eigenthümlich dreikantig. Wimpern waren nicht erhalten, werden aber im lebenden Zustand kaum gefehlt haben.

Ganglion und benachbarte Organe. Das sogen. Gehirnganglion, die Neuraldrüse und der Rückenhöcker stehen in so enger Verbindung, dass ihre gemeinsame Behandlung sich rechtfertigt. Letztere beiden theilen übrigens das Schicksal, dass sie sich die verschiedenartigsten Deutungen haben gefallen lassen müssen.

Das Ganglion befindet sich in der von allen Synascidien bekannten Lage in der Mittellinie des Körpers zwischen Einfuhr- und Ausfuhröffnung, jedoch mehr der ersteren genähert und unmittelbar unter dem Muskelmantel. Es ist von ovaler Form (Fig. 4, 5 u. 12 GG) und zeigt, wie immer, eine zell- und kernreichere Peripherie und eine daran ärmere centrale Masse. Die an beiden Polen abgehenden Stämme lassen sich nur eine ganz kurze Strecke weit verfolgen. Man weiss von günstigeren Objecten, dass sie einerseits gegen den Kiemensack, andrerseits gegen die Peripharyngealbänder und den Tentakelkranz hin sich fortsetzen. Die Angabe GIARD'S ${ }^{1}$ ), dass eine Art Schlundring vorhanden sei, wird von Della V VLLe ${ }^{2}$ ) als wahrscheinlich unzutreffend erklärt, wie denn überhaupt in dieser Frage noch grosse Unsicherheit besteht. Die Ausbildung einer Methode, welche bei Wirbellosen die specifische Färbung der (marklosen) Nervenfasern in jener vollendeten Weise gestattete, welche das WeIGERT'sche Hämatoxylin-KupferlackVerfahren bei den (markhaltigen) Fasern der Wirbelthiere ermöglichte, würde einen gewaltigen Fortschritt bedeuten.

Die sogen. Neuraldrüse liegt unmittelbar unter dem Ganglion.

1) GIARD, 1. c. p. 514.

2) Della Valle, l. c. p. 42. 
Häufig von Eiform, stellt sie hier eine fast kuglige Masse dar (Fig. 4 u. 12 ND). Der nach vorn bezw. oben abgehende Ausführungsgang beginnt noch zwischen ihr und dem Ganglion, tritt dann aber unter demselben als kurze, gegen das Ende erweiterte Röhre hervor. Sie mündet mit enger Kreisöffnung an der Stelle, wo die beiden seitlichen Bogen des Peripharyngealbandes dorsal auf einem gegen den Kiemensack zu gewölbten Vorsprung zusammentreffen, welcher wie jene Wimperzellen trägt. Die genaue, bei zahlreichen Exemplaren in derselben Weise gesehene Gestalt dieses Tuberkels ist in Figur 12 abgebildet. Das Ascidiozooid lag ungefähr symmetrisch zu dem in Fig. 4 wiedergegebenen, und der Beobachter, welcher das Organ in der in Fig. 12 gezeichneten Ansicht erblicken möchte, müsste etwa am unteren Ende des Endostyls stehen. Bei Vergleichung anderer genau untersuchter Arten erkennt man selbst an diesen kleinen Gebilden mehrfache Abweichungen. In der Deutung dieses Organes und der sogen. Neuraldrüse nicht minder, gehen, wie gesagt, die Meinungen weit auseinander. Während Herdman dafür hält ${ }^{1}$ ), dass die Untersuchungen von Ussow und Julin die Unabhängigkeit beider vom Gehirnganglion erwiesen und die frühere Deutung als Geruchsorgan beseitigt hätten, betont Della Valle zu ungefähr gleicher Zeit auf Grund entwicklungsgeschichtlicher Studien die ursprünglich nervöse Natur ${ }^{2}$ ). Auf Grund der Untersuchung einer einzigen Art ist selbstverständlich kein Urtheil möglich.

Der Verda u ungs apparat. Die Eingangsöffnung zum eigentlichen Verdauungsapparat nimmt den etwas verschmälerten Grund des Kiemensackes fast zur Hälfte ein. Sie besitzt eine kleine aufgewulstete Lippe (Fig. 4 bei $O e O e$ ). Dieselbe führt in einen engeren, meist gerade nach abwärts verlaufenden, bisweilen aber auch in kurze, dicht aufeinanderfolgende Windungen gelegten Oesophagus. Der Magen, welcher ebenfalls die Längsrichtung der Speiseröhre beibehält, ist eine sehr ansehnliche, kuglige oder eiförmige Erweiterung, die sich durch ihre auch innerlich ausgeprägte Längsfaltung noch besonders auszeichnet (vergl. Fig. 4 u. $6 M$ ). Da sowohl die Speiseröhre wie der Darm die Magenwandung gleichsam etwas gegen das Lumen des Magens $\mathrm{zu}$ einstülpt, und die Längsfurchen ziemlich tief sind, gehen die Falten, 6-10 an Zahl, oft in förmliche Aussackungen über. Es tritt dies

1) Herdman, 1. c. part I, p. 45.

2) Deflia Valie, l. c. p. 44. 
namentlich hervor, wenn ihre stärksten Wölbungen nicht in der Mitte liegen, sondern nach oben gerückt sind und andrerseits die Furchen das untere Ende des Magens nicht erreichen - ein auch in der Zeichnung dargestellter Fall. Auf dem Querschnitt erkennt man die Faltung natürlich sofort (Fig. $6 M$ ) und im weiteren, dass die ziemlich dicken Wände aus einer einzigen Schicht hoher Cylinderzellen gebildet werden. Von oben gesehen, setzen diese Zellen ein zierliches polygonales Mosaik zusammen. Aehnlich gefaltet ist die Magenwand nach den bisher veröffentlichten Angaben bei Botryllus, Aplidium, Amaroucium, Tylobranchion und Goodsiria.

Der Darm zeigt einige kurze Windungen (Fig. 4, DD), welche über und neben dem, den Grund des Eingeweidesackes einnehmenden Hoden liegen. Nach scharfer Umbiegung und unter bedeutender Erweiterung steigt er als dünnwandiges Rectum wieder nach aufwärts, gewöhnlich an derselben Seite, wo er abstieg. Meist ist er mit einer Menge von Nahrungsresten gefüllt, welche eiförmige Ballen bilden. Neben allerlei Resten unbestimmbarer Herkunft erblickt man zahlreiche Spongiennadeln, Diatomeenschalen u. dergl. mehr. Ungefähr in der Mitte der Peribranchialhöhle verengert sich der Darm wieder etwas und endigt hier mit einer von vier schmalen Zipfeln umstellten Oeffinung, der eigentlichen Afteröffnung (Fig. $4 A$ ). - Das System verzweigter feiner Schläuche, welches bei vielen Synascidien einen Theil des Magens oder Darms überzieht und Verdauungsdrüse kurzweg oder unter mehr functioneller Bezeichnung Glandula hepato-pancreatica genannt wurde, kounte ich an Uebersichtspräparaten nicht nachweisen. Dagegen sah ich an Schnitten mehrmals Bilder, wie Fig. 7 (bei $L$ ) eines darstellt. Es entspricht dem Della Valle'schen Durchschnitt der Leberschläuche von Botryllus aurolineatus ${ }^{1}$ ) und lässt also auf die Anwesenheit der erwähnten Drüsenformation auch bei unserer Art schliessen.

Bezüglich des Circulationsapparates ist nur zu bemerken, dass das Herz seine gewöhnliche Lage in der Längsrichtung des Körpers neben dem Verdauungstractus innehält.

Der Geschlechtsapparat. Die Synascidien sind bekanntlich Zwitter. Da indessen die beiderlei Geschlechtsorgane und ihre Producte sich ungleichzeitig, jedoch bei allen Thieren einer Colonie gleich rasch entwickeln, so findet man entweder stets rein männliche oder rein weibliche Colonieen mit lauter ungefähr gleich weit gereifte

1) Delua Valle, 1. c. T. II, Fig. 20. 
Thieren. Die vorliegende Colonie war eine rein männliche; vom Eierstock liess sich keine Spur erkennen.

Der Hoden besteht aus $8-16$ rundlichen Testikeln von $0,05-0,07 \mathrm{~mm}$ Durchmesser. Dieselben nehmen den untersten Theil des Eingeweidesackes ein, ihre feinen Ausführungsgänge jedoch wenden sich alle in kürzerem oder längerem Bogen dorsalwärts, um hier, fast in demselben Punkt, zu einem Samenleiter, einem Vas deferens, zusammenzutreten. Betrachtet man die Gruppe der Testikel von der Dorsalseite her, so scheinen sie radiär um ein gemeinsames Centrum geordnet zu sein, wie das z. B. von Drasche ${ }^{1}$ ) für Distomiden und Distapliden beschreibt und abbildet. In den Testikeln sind kleine, mit stark färbbaren Kernen versehene Zellen eng gehäuft. Die mehr dem äusseren Umkreis jedes Testikels genäherten sind etwas grösser, und ihre Kerne sind etwas blässer als die näher der Mitte befindlichen. In der Mitte selbst kann man oft dichtgedrängte Mengen fertig ausgebildeter Spermatozooen wahrnehmen. Danach sind auch hier, an der Peripherie beginnende und centripetal fortschreitende, wiederholte Kern- und Zelltheilungsprocesse als Vorstufen der Spermatozoenbildung vorauszusetzen. Die Spermatozoen selbst stellen gleichmässig dünne, wellenförmig gebogene Fädchen dar, deren Geisseln nicht mehr sichtbar zu machen waren.

Der Verlauf des Samenleiters ist leicht zu verfolgen, da er gewöhnlich voll Spermatozoen gepfropft ist und sich darum sehr dunkel färbt, während die Faeces des theilweise darunter liegenden Darmes ungefärbt, bezw. hellbraun bleiben. Der Samenleiter tritt nach leicht bogenförmigem, wohl auch noch einige stärkere Krümmungen einschliessendem Verlauf (Fig. $4 S$ ) in den Peribranchialraum ein, schwillt hier leicht und allmählich an, um endlich, unter ebenso allmählicher Verengerung seines Lumens, neben der Afteröffnung in diesen Peribranchialraum auszumünden.

Der Peribranchial - oder Cloakenraum ist jener Raum, welcher einerseits das aus dem Kiemensack abfliessende Wasser, andrerseits die aus den Organen des Eingeweidesackes stammenden Nahrungsreste und Geschlechtsproducte aufnimmt und durch die Anal- oder Ausfuhröffnung nach aussen abgiebt. Er hat seine grösste Weite dorsal vom Kiemensack, umgreift jedoch auch, obschon bedeutend verschmälert, die beiden Seitenflächen desselben. Bei unserer Art ist die

1) R. v. Drasche, Die Synascidien der Bucht von Rovigno, Wien 1883 , p. 16 ff., Taf. IX, Fig. 1. 
bauchige Anschwellung des Cloakenraumes ziemlich stark; [in der Längsrichtung reicht er bis etwa zum letzten Drittel der Höhe des Kiemensackes. Die Eigenthümlichkeiten seiner Oeffnung wurden schon früher erwähnt (p. 867).

Systematische Stellung. Gegenüber der äusseren Form der Gesammtcolonie, welche selbst bei Synascidien gleicher Art in hohem Grade wechseln kann, liefert der Bau der Einzelthiere die wichtigsten systematisch verwendbaren Charactere. Dass unsere Form auf Grund dessen zunächst in die Milne Edwards'sche Gruppe der „Didemniens" gehört, wurde bereits früher festgestellt. Da jedoch die spätere Forschung zeigte, dass die genannte Gruppe keine so natürliche ist, wie die gleichzeitig und nach denselben Grundsätzen aufgestellte der „Polycliniens" und „Botrylliens", so wurde sie in eine Anzahl von Familien aufgelöst, welche bei der Erörterung der systematischen Stellung unserer Art zu berücksichtigen sein werden.

Folge ich der Eintheilung, welche Herdman 1886 der umfassenden Bearbeitung der Challenger-Synascidien zu Grunde legte, so kommen die älteren Familien der Distomidae, Didemnidae und Diplosomidae und zwei ausschliesslich auf Challenger-Material begründete neue Familien in Betracht. Von den Didemnidae und Diplosomidae weicht unsere Form unter anderem durch die viel grössere Zahl von Kiemenreihen, von den Challenger-Familien der Coelocormidae und Polystyelidae durch den sechs-(statt fünf-, bezw. vier-)zähnigen Einfuhrtrichter und viele andere Merkmale ab. Dagegen vereinigt die Form alle Charactere auf sich, welche die Familie der Distomidae auszeichnen, und zwar in dem ihr von Herdman gegebenen, die Familie der Chondrostachyidae vON Drasche's einschliessenden Umfang.

Die (nachstehend durch gesperrten Druck ausgezeichneten) Merkmale der Herdman'schen Distomidae ${ }^{2}$ ) kommen, wie aus den vorausgegangenen Schilderungen hervorgeht, sämmtlich auch unserer Form zu.

\section{Familie Distomidae.}

Colonie abgerundet und massig, selten Krusten bildend, sitzend oder von einem längeren oder kürzeren Stiel getragen. fehlend.

Systeme unregelmässig, wenig hervortretend oder

1) Herdman, 1. c. part II.

2) Herdman, l. ult. c. p. 64. 
Ascidiozooiden von mässiger Länge, mit zwei Körperregionen; können mit langen Ektodermfortsätzen versehen sein.

Testa gelatinös oder knorpelartig, am Grund oft zu einem Stiel verdickt ....

Kiemensack gut entwickelt, gewöhnlich ohne innere Läng sleis ten.

Lamina dorsalis in Form von Languets, selten eine einfache Membran.

Nahrungscanal am hinteren Ende des Kiemensackes gelegen.

Fortpflanzungsorgane im Eingeweidesack oder neben demselben.

Prüfen wir nun die sieben Genera der Distomidae, so steht $D i$ stoma unserer Form unzweifelhaft am nächsten. Symplegma besitzt innere Längsfalten, welche hier fehlen. Chondrostachys, Oxycorynia, Colella sind schon durch den deutlich entwickelten Stiel ausgeschlossen. Von Cystodytes unterscheidet sich unsere Form durch die Abwesenheit von Kalkhüllen um die Einzelthiere, von Distaplia durch die weitaus grössere Zahl von Kiemenreihen.

Stimmt aber die Form auch am besten mit Distoma überein, so unterscheidet sie sich doch von den Angehörigen auch dieser Gattung durch die abweichende Gestalt und Umgebung der Ausfuhröffnung. von Drasche ${ }^{1}$ ) characterisirt das Genus Distoma wie folgt:

„Ig. Oe. (Ingestionsöffnung) und Eg. Oe. (Egestionsöffnung) auf langen sechszähnigen Trichtern, Eingeweidesack oft gestielt und dann beträgt die Länge desselben oft das Vielfache des Thorax."

Wie wir sahen, ist bei unserer Form nur die Einfuhröffnung von einem sechszähnigen Trichter, die Ausfuhröffnung aber einfach von einer glatt begrenzten Ringmuskellage umschlossen. Dagegen tritt über ihr eine dreispaltige Analzunge auf, welche einerseits Distoma fehlt, andrerseits, wenn auch in abweichender Art, bei Distaplia sich findet $^{2}$ ).

Dies alles dürfte zur Aufstellung eines neuen Genus nöthigen,

1) v. Drasche, 1. c. p. 17.

2) Die Abwesenheit eines Brutraumes für die Eier, eines „incubatory pouch", wie er Distaplia zukommt, kann natürlich nicht sicher behauptet werden, wegen des rein männlichen Characters der Colonie. Indessen erlauben schon die übrigen Abweichungen einen Anschluss an Distaplia nicht. 
welches zwischen Distoma und Distaplia, jedoch in näherem Anschluss an Distoma, in die Familie der Distomidae einzureihen wäre.

In Berücksichtigung der zuletzt erwähnten Merkmale und zu Ehren der Entdecker möchte ich den Namen

\section{Heterotrema sarasinorum}

für diese neue Form in Vorschlag bringen.

Das neue Genus, mit der einzigen bisher dahin gehörigen Species, kann folgendermaassen characterisirt werden:

Colonie ansehnliche, plump verzweigte, rundliche Massen bildend, die an mehreren Stellen mit dem Untergrunde verwachsen sind.

Systeme wenig hervortretend, von seichten Furchen begrenzt, welche unregelmässige polygonale Maschen bilden.

Testa gelatinös.

Einzelthiere $2-3 \mathrm{~mm}$ lang, mit zwei Körperabschnitten und Ectodermfortsätzen.

Branchialöffnung mit sechszähnigem Trichter, Analöffnung ein einfacher Schlitz, mit dreizipfligem Analanhang.

Kiemensack gut entwickelt, ohne innere Längsleisten, mit 8-10 Reihen von Kiemenöffnungen.

Lamina dorsalis in Form von Languets.

Nahrungscanal am hinteren Ende des Kiemensackes gelegen, Magen mit Längsfalten.

Hoden, aus zahlreichen, traubig angeordneten Follikeln bestehend, am Grunde des Eingeweidesackes.

Zürich-Hottingen, 1. Juli 1889. 


\section{Figuren-Erklärung.}

\section{Tafel XXV.}

Allgemein gültige Bezeichnungen (alphabetisch geordnet): $A$ Afteröffnung, $A A$ Analanhänge, $A$. Oe Ausfuhröffnung, $D D$ Darm, DT Dorsal-Tuberkel, $E$ Endostyl, E. Oe Einfuhröffnung, G Gefässfortsatz, - $G G$ Gehirnganglion, $H$ Hoden, $L$ Languets, $L L$ Leberschläuche, $M$ Magen, $M D$ Mastdarm, $M F$ Muskelfortsatz, $N$ ? Nierenzellen (?), $N D$ Neuraldrüse, Oe. Oe Oesophagealöffnung, $P B$ Peripharyngealband, $S$ Samenleiter, $T$ Tentakel.

Fig. 1. Colonie von Heterotrema sarasinorum in natürlicher Grösse.

Fig. 2. Querschnitt eines Zweiges der Colonie: Anordnung der Einzelthiere. Natürliche Grösse.

Fig. 3. Theil eines Querschnittes der Colonie: Verbindung von Einfuhröffnung und Mantelmasse, Anal- und Gefässfortsatz. Vergr. 75.

Fig. 4. Einzelthier: allgemeine Uebersicht. Vergr. 75.

Fig. 5. Einfuhröffnung von oben: Anordnung der Muskelbänder. Vergröss. 120.

Fig. 6 и. 7. Querschnitte durch den Eingeweidesack eines (kleineren) Einzelthieres, Magen, Leberschläuche. Vergr. 75.

Fig. 8. Querschnitt des Endostyls von Polycyclus renieri nach DewuA VALLE.

Fig. 9. Querschnitt des Endostyls von Botrylloides perspicuum nach HERdMAN.

Fig. 10. Querschnitt des Endostyls von Heterotrema sarasinorum. Vergröss. 300.

Fig. 11. Endostyl von Heterotrema sarasinorum, Flächenansicht. Vergr. 120.

Fig. 13. Blasen-, Kugel- oder Hohlzellen der gemeinsamen Mantelmasse. Vergr. 650 .

Fig. 14. Wimperzellen des Kiemensackes. Vergr. 650. 


\section{$2 \mathrm{BHL}$ Biodiversity Heritage Library}

Fiedler, Karl. 1889. "Heterotrema sarasinorum, eine neue Synascidien Gattung aus der Familie der Distomidae." Zoologische Jahrbücher 4, 859-878. https://doi.org/10.5962/bhl.part.18730.

View This Item Online: https://www.biodiversitylibrary.org/item/38153

DOI: https://doi.org/10.5962/bhl.part.18730

Permalink: https://www.biodiversitylibrary.org/partpdf/18730

\section{Holding Institution}

MBLWHOI Library

\section{Sponsored by}

MBLWHOI Library

\section{Copyright \& Reuse}

Copyright Status: NOT_IN_COPYRIGHT

This document was created from content at the Biodiversity Heritage Library, the world's largest open access digital library for biodiversity literature and archives. Visit BHL at https://www.biodiversitylibrary.org. 